\title{
openheart Pre-hospital One-Hour Troponin in a Low-Prevalence Population of Acute Coronary Syndrome: OUT-ACS study
}

\author{
Tonje R Johannessen (D) ,,2 Odd Martin Vallersnes (D) ,1,2 Sigrun Halvorsen (D) ,3,4 \\ Anne Cecilie K. Larstorp, ${ }^{4,5}$ Ibrahimu Mdala (D) , ${ }^{1}$ Dan Atar (1) ${ }^{3,4}$
}

\begin{abstract}
- Additional material is published online only. To view, please visit the journal online (http://dx.doi.org/10.1136/ openhrt-2020-001296).
\end{abstract}

To cite: Johannessen TR, Vallersnes OM, Halvorsen S, et al. Pre-hospital One-Hour Troponin in a Low-Prevalence Population of Acute Coronary Syndrome: OUT-ACS study. Open Heart 2020;7:e001296. doi:10.1136/

openhrt-2020-001296

Received 27 March 2020 Revised 8 May 2020 Accepted 26 May 2020

Check for updates

(c) Author(s) (or their employer(s)) 2020. Re-use permitted under CC BY-NC. No commercial re-use. See rights and permissions. Published by BMJ.

'Department of General Practice Institute of Health and Society, University of Oslo, Oslo, Norway ${ }^{2}$ Oslo Accident and Emergency Outpatient Clinic, City of Oslo Health Services, Oslo, Norway ${ }^{3}$ Department of Cardiology, 0slo University Hospital Ullevaal, Oslo, Norway

${ }^{4}$ Institute of Clinical Medicine, University of Oslo, Oslo, Norway ${ }^{5}$ Department of Medical Biochemistry, Section of Cardiovascular and Renal Reseach, Oslo University Hospital Ullevaal, Oslo, Norway

Correspondence to Dr Tonje R Johannessen; t.r. johannessen@medisin.uio.no

\section{ABSTRACT}

Objective The European Society of Cardiology 0/1-hour algorithm for high-sensitivity cardiac troponin T (hs-cTnT) has demonstrated high rule-out safety in large hospital validation cohorts. We aimed to validate the algorithm in a primary care setting, where patients have a lower pretest probability for acute coronary syndrome.

Methods This prospective, observational, diagnostic study included patients with acute non-specific chest pain admitted to a primary care emergency clinic in Oslo, Norway, from November 2016 to October 2018. hscTnT was measured after 0,1 and 4 hours. The primary outcome measure was the diagnostic performance of the 0/1-hour algorithm, the 90-day incidence of AMI or allcause death the secondary.

Results Among 1711 included patients, 61 (3.6\%) were diagnosed with AMI. By applying the algorithm, $1311(76.6 \%)$ patients were assigned to the rule-out group. The negative predictive value was $99.9 \%(95 \% \mathrm{Cl}$ $99.5 \%$ to $100.0 \%$ ), the sensitivity and specificity $98.4 \%$ (91.2-100.0) and $79.4 \%$ (77.4-81.3), respectively. Sixtysix $(3.9 \%)$ patients were triaged towards rule-in, where 45 were diagnosed with AMI. The corresponding positive predictive value was $68.2 \%$ (58.3-76.7), sensitivity $73.8 \%$ (60.9-84.2), and specificity $98.7 \%$ (98.1-99.2). Among $334(19.5 \%)$ patients assigned to the observation group in need of further tests, 15 patients had an AMI. The following 90 days, five new patients experienced an AMI and nine patients died, with a low incidence in the rule-out group $(0.3 \%)$.

Conclusion The 0/1-hour algorithm for hs-cTnT seems safe, efficient and applicable for an accelerated assessment of patients with non-specific chest pain in a primary care emergency setting.

Trial registration number NCT02983123.

\section{INTRODUCTION}

Rapid triage of suspected acute coronary syndrome (ACS) is crucial in patients presenting with acute chest pain. In addition to clinical assessment and the ECG, cardiac troponins are gold standard biomarkers in the diagnosis of acute myocardial infarction (AMI) ${ }^{12}$ Due to limited diagnostic tests, the AMI diagnosis is challenging in the prehospital emergency setting, ${ }^{3-5}$ and the value of

\section{Key questions}

What is already known about this subject?

- Ruling out acute myocardial infarction in primary care is challenging due to limited diagnostic decision aids. The favourable diagnostic performance of the 0/1-hour algorithm for high-sensitivity cardiac troponins has earlier been validated in hospital studies, with high rule-out safety and efficacy.

What does this study add?

- In this observational diagnostic study, the same algorithm seems safe, efficient and accurate, also in a primary care emergency setting, where the patients with acute chest pain have a lower pretest probability for acute coronary syndrome.

How might this impact on clinical practice?

- By implementing this algorithm for rapid and safe triage done by general practitioners outside of hospitals, the overall costs, the risk of overdiagnosis, and patient crowding in the emergency departments may be reduced.

prehospital risk stratification with point-ofcare troponins with or without risk assessment scores has received increased attention during the last decade. ${ }^{6-8}$ Still, there is no prehospital strategy that safely excludes AMI outside of hospitals. ${ }^{589}$

The introduction of high-sensitivity assays for cardiac troponins opened for rapid diagnostic pathways in hospitals, ${ }^{10-12}$ and the diagnostic utility of the 2015 European Society of Cardiology (ESC) 0/1-hour algorithm for high-sensitivity cardiac troponin $\mathrm{T}$ (hs-cTnT) ${ }^{2}$ has been confirmed in large validation studies from hospital emergency departments (EDs). ${ }^{13-18}$ However, there is a need for validation of the algorithm also in a primary care setting, where the patients have a lower pretest probability for ACS. ${ }^{13} 141618$

We aimed to validate the 0/1-hour algorithm for hs-cTnT in a low-prevalence population for ACS by applying the algorithm in a primary care emergency setting. 
Furthermore, we registered the incidence of new AMIs or all-cause deaths during the 90 days following the initial assessment.

\section{METHODS}

\section{Study design and setting}

The One-hoUr Troponin in a low-prevalence population of Acute Coronary Syndrome or OUT-ACS study is a single-centre, observational, prospective, diagnostic cohort study, conducted at Oslo Accident and Emergency Outpatient Clinic (OAEOC) in Norway. The OAEOC is the main primary care emergency outpatient clinic in Oslo, with approximately 200000 consultations per year and has an observation unit with 18 beds. The OAEOC serves the entire city (681 071 inhabitants as per 1 January 2019) ${ }^{19} 24 / 7$ all year.

The emergency care system in Norway is two-tiered, with an active gatekeeping function in primary care, regulating access to the hospitals. Hence, patients with acute symptoms are initially assessed outside of hospital. Patients considered critically ill (approximately 50\% of all AMIs) bypass the gatekeeping system and are directly brought to hospital by ambulance services. ${ }^{20}$ The remaining patients are treated in primary care or sent on to the hospital after primary care assessment. The primary care emergency clinics differ from hospital EDs by having less advanced diagnostic resources and therapeutic options and are mainly staffed by general practitioners (GPs).

\section{Participants}

During the enrolment period (November 2016-October 2018), the study consecutively recruited patients, 18 years or older, with non-traumatic chest pain or discomfort admitted to the prehospital OAEOC observation unit for assessment of cardiac troponins (figure 1). Patients admitted for cardiac troponin measurements after electric trauma were not included, nor were patients with a highly suspected ACS (comprising AMIs with or without ST-segment elevations, and unstable angina pectoris), as they were rapidly sent on to the hospital after initial assessment by the GP.

\section{Data collection}

The GP obtained a medical history and performed a physical examination of all patients presenting with chest complaints, including pulse oximetry and ECG. Capillary $\mathrm{C}$ reactive protein, haemoglobin, blood glucose and chest X-ray were the only additional tests available. Whether the patient was directly hospitalised due to a suspected ACS, sent home with no additional tests or admitted to the prehospital observation unit for cardiac troponins was left to the discretion of the individual GP, following regular practice at the clinic. Further details are illustrated in online supplementary figure $\mathrm{S} 1$ in appendix.

The 0-hour hs-cTnT was sampled immediately after admission to the observation unit. The 1-hour study sample was drawn by the regular nursing staff after written informed consent was obtained. Details regarding risk factors, symptom presentation and time intervals were recorded in a predefined form. In addition, the regular 4-hour hs-cTnT, kidney function tests and additional ECGs were collected, and hospital discharge documents were gathered from all hospitalised participants.

New incidents of AMI or all-cause death the following 90 days were obtained through linkage with the Norwegian Cardiovascular Disease Registry. ${ }^{21}$ This national register gathers data from the Norwegian Patient Registry, the Norwegian Cause of Death Registry and the Norwegian Central Population Registry. In addition, cardiovascular codes from the International Statistical Classification of Diseases and Related Health Problems, 10th Revision (ICD-10), ${ }^{22}$ are automatically reported to the Norwegian Cardiovascular Disease Registry after hospital admissions and hospital outpatient clinic visits. ${ }^{23}$ For this study, we extracted primary and secondary ICD-10 chapter I21-22 (AMI) codes, date of the incidence and date of death.

\section{Laboratory analysis}

Following the standard procedure at the OAEOC, venous blood samples were collected in serum tubes and stored locally at room temperature (approximately $20^{\circ} \mathrm{C}$ ) for a maximum of $30 \mathrm{~min}$ before centrifugation. The serum was stored in a refrigerator before being sent on to the Central Lab at Oslo University Hospital Ullevaal for analysis every 4 hours. The 1-hour samples were collected 55-90 min after the 0-hour sample. hs-cTnT was analysed on the Cobas 8000 e602 and later the Cobas 8000 e801 Module Analyzer using the Elecsys Troponin $\mathrm{T}$ hs STAT assay (Roche Diagnostics, Switzerland). For hs-cTnT, the 99th percentile of a healthy reference population is $14 \mathrm{ng} / \mathrm{L}$, with a coefficient of variation (CV) of $<10 \%$, a limit of detection of $5 \mathrm{ng} / \mathrm{L}$, a limit of blank of $3 \mathrm{ng} / \mathrm{L}$ and stability of cTnT with storage at $2^{\circ} \mathrm{C}-8^{\circ} \mathrm{C}$ of 24 hours. ${ }^{24} 25$ A stability of 24 hours has also been demonstrated for samples stored under the conditions in our study. ${ }^{26}$ During the study period, the laboratory regularly analysed EQA (external quality assessment) material from Noklus (Bergen, Norway) and Equalis (Equalis AB, Uppsala, Sweden) with good performance. The CV was $10 \%$ at concentrations of $<20 \mathrm{ng} / \mathrm{L}$ and $6 \%$ at concentrations of $\geq 20 \mathrm{ng} / \mathrm{L}$.

\section{The 0/1-hour algorithm for hs-cTnT}

The 0/1-hour rule-in/rule-out algorithm for hs-cTnT follows assay-specific cut-off values ${ }^{13}$ as described in the 2015 ESC guidelines on non-ST-elevation myocardial infarction. ${ }^{2}$ Patients are classified into rule-out, rule-in or further observation, according to the 0-hour (0h) hs-cTnT sample alone, or the absolute $0-1$ hour change $(\Delta 0-1 \mathrm{~h})$ (figure 2). During the study, the 1-hour hs-cTnT measurement was available to the GP treating the patient at the observation unit to avoid a prehospital delay among patients assigned towards rule-in by the 1-hour sample. 


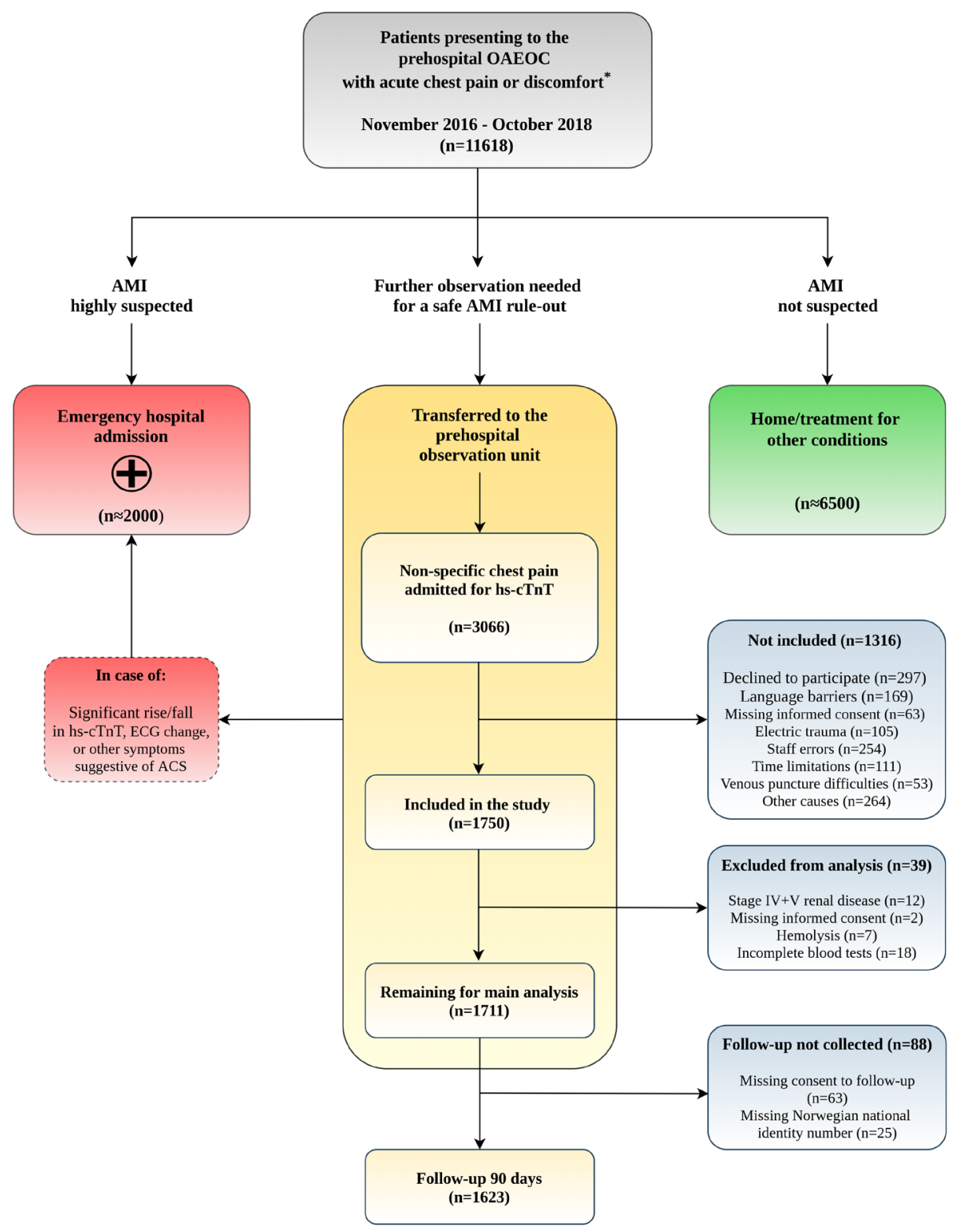

Figure 1 Patient flow diagram. Management of acute chest pain at the OAEOC and patient flowchart during the study. *, critically ill patients are directly hospitalised by the ambulance services. ACS, acute coronary syndrome; AMI, acute myocardial infarction; hs-cTnT, high-sensitivity cardiac troponin T; OAEOC, Oslo Accident and Emergency Outpatient Clinic.

\section{Final diagnosis}

In addition to the clinical assessment and the repeated ECGs, the standard hs-cTnT of $\Delta 0-4$ hours served as a reference standard for ruling out AMI for all patients discharged home. The treating GP interpreted the $\Delta 0-4$ hours according to the 'Third Universal Definition of Myocardial Infarction' (applicable at the time of the study), comprising a significant rise/fall pattern of hs-cTnT with at least one value above the 99th percentile of a healthy reference population, in combination with ischaemic symptoms, or pathological ECG changes. For baseline values above the 99th percentile, a relative change of $20 \%$ or more was considered significant; for baseline values below the 99th percentile, the relative change had to be at least $50 \%{ }^{1}$
Two independent cardiologists at Oslo University Hospital adjudicated the final AMI diagnosis for all hospitalised patients, with access to all collected data from both the OAEOC and the hospital admission during the index episode, including the 1-hour hs-cTnT measurement. The adjudication process was based on the 'Third Universal Definition of Myocardial Infarction'. ${ }^{1}$ A third cardiologist was consulted if there was any disagreement in the adjudication (in 19 of the cases).

\section{Outcome measures}

The primary outcome of the study was the diagnostic performance of the 0/1-hour algorithm for AMI at the index episode, and the safety in the rule-out group, as measured 


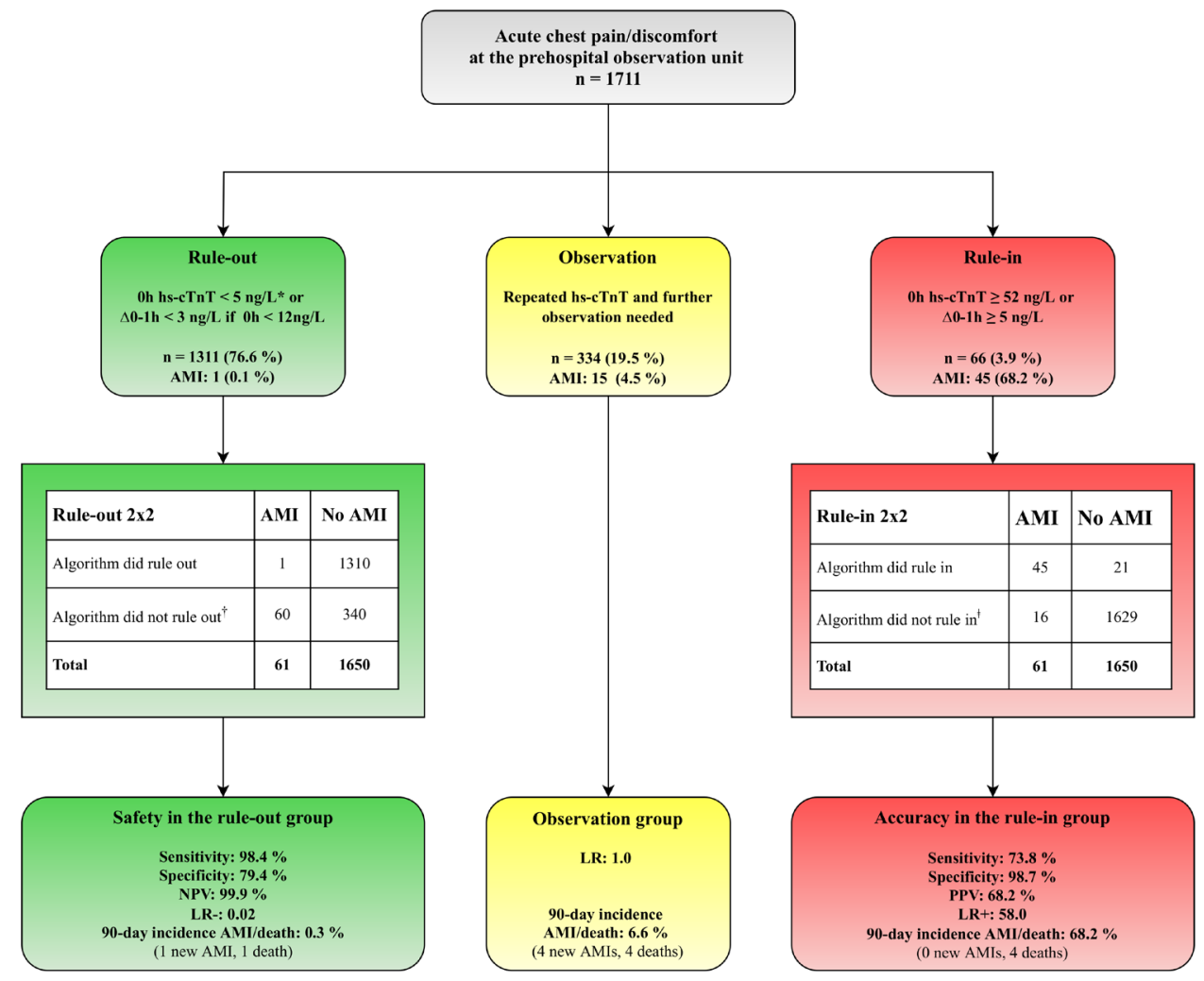

by the negative predictive value (NPV) and the sensitivity. The index episode was defined as the event resulting in prehospital hs-cTnT sampling. In the rule-in group, we measured the diagnostic accuracy (the positive predictive value (PPV) and the specificity) to address whether the algorithm resulted in too many false positives when applied in a low-prevalence setting.

Secondary outcome measures were AMI (including the adjudicated AMI at the index episode) or all-cause death during the subsequent 90 days as a prognostic evaluation of the algorithm. In addition, the proportion of patients correctly triaged by the 0/1-hour algorithm (ruled-out without AMI or ruled-in with AMI) and the overall efficacy, that is, the proportion of patients assigned to either the rule-out or the rule-in group, were estimated.

\section{Statistical analysis}

The categorical variables are presented as numbers and percentages; the continuous variables are presented as medians and IQRs. Comparisons of categorical variables were made using the Pearson $\chi^{2}$ test or the Fisher exact test, whereas the Kruskal-Wallis test was used when comparing continuous variables. We used two-sided hypothesis testing, and the significance level was set at $\alpha=0.05$. The sample size calculation is described in detail in the online supplementary appendix.

Since the 0/1-hour algorithm has three outcomes (rule-out, rule-in and observation), it does not provide a dichotomic positive/negative test result. The diagnostic performance of the algorithm is, therefore, calculated for the rule-out and the rule-in groups separately. In 
Coronary artery disease

\begin{tabular}{|c|c|c|c|c|c|}
\hline & $\begin{array}{l}\text { Total } \\
n=1711\end{array}$ & $\begin{array}{l}\text { Rule-out } \\
n=1311\end{array}$ & $\begin{array}{l}\text { Observation } \\
n=334\end{array}$ & $\begin{array}{l}\text { Rule-in } \\
n=66\end{array}$ & $P$ value \\
\hline Female sex, $\mathrm{n}(\%)$ & $816(47.7)$ & $640(48.8)$ & $150(44.9)$ & $26(39.4)$ & 0.177 \\
\hline Age, median (IQR) & $56(45-68)$ & $52(42-62)$ & $72(62-83)$ & $65(53-82.3)$ & $<0.001$ \\
\hline \multicolumn{6}{|l|}{ Risk factors for CVD, $\mathrm{n}(\%)$} \\
\hline Current/history of smoking & $449(26.2)$ & $368(28.1)$ & $61(18.3)$ & $20(30.3)$ & 0.001 \\
\hline Previous coronary artery disease & $317(18.5)$ & $165(12.6)$ & $135(40.4)$ & $17(25.8)$ & $<0.001$ \\
\hline Hypertension & 448 (26.2) & $293(22.3)$ & 139 (41.6) & $16(24.2)$ & $<0.001$ \\
\hline Hypercholesterolaemia & $422(24.7)$ & $295(22.5)$ & $110(32.9)$ & $17(25.8)$ & $<0.001$ \\
\hline Other CVD* & $288(16.8)$ & $146(11.1)$ & $123(36.8)$ & $19(28.8)$ & $<0.001$ \\
\hline Diabetes mellitus & $171(10.0)$ & $106(8.1)$ & $55(16.5)$ & $10(15.2)$ & $<0.001$ \\
\hline COPD & $80(4.7)$ & $38(2.9)$ & $37(11.1)$ & $5(7.6)$ & $<0.001$ \\
\hline Family history of CVD & $690(40.3)$ & $564(43.0)$ & $101(30.2)$ & $25(37.9)$ & $<0.001$ \\
\hline \multicolumn{6}{|l|}{ Presenting acute symptoms, $\mathrm{n}(\%)$} \\
\hline Chest pain & $1485(86.8)$ & $1174(89.5)$ & $252(75.4)$ & $59(89.4)$ & $<0.001$ \\
\hline Constricting & $1239(72.4)$ & $978(74.6)$ & $206(61.7)$ & $55(83.3)$ & $<0.001$ \\
\hline Sharp & $404(23.6)$ & $339(25.9)$ & $57(17.1)$ & $8(12.1)$ & $<0.001$ \\
\hline Tearing & $64(3.7)$ & $54(4.1)$ & $7(2.1)$ & $3(4.5)$ & 0.157 \\
\hline Burning & $208(12.2)$ & $166(12.7)$ & $32(9.6)$ & $10(15.2)$ & 0.226 \\
\hline Respiratory dependent & $302(17.7)$ & $250(19.1)$ & $41(12.3)$ & $11(16.7)$ & 0.014 \\
\hline Chest-wall tenderness & $205(12.0)$ & $170(13.0)$ & $33(9.9)$ & $2(3.0)$ & 0.022 \\
\hline Movement dependent & $219(12.8)$ & $183(14.0)$ & $35(10.5)$ & $1(1.5)$ & 0.005 \\
\hline Other pain (abdomen, back or neck) & $48(2.8)$ & $32(2.4)$ & $14(4.2)$ & $2(3.0)$ & 0.175 \\
\hline No pain & $177(10.3)$ & $104(7.9)$ & $68(20.4)$ & $5(7.6)$ & $<0.001$ \\
\hline Pain radiation & $1000(58.4)$ & $802(61.2)$ & $154(46.1)$ & $44(66.7)$ & $<0.001$ \\
\hline Dyspnoea & $901(52.7)$ & $689(52.6)$ & $178(53.3)$ & $34(51.5)$ & 0.962 \\
\hline Palpitations & $637(37.2)$ & $501(38.2)$ & $117(35.0)$ & $19(28.8)$ & 0.195 \\
\hline Syncope/presyncope & $460(26.9)$ & $353(26.9)$ & $88(26.3)$ & $19(28.8)$ & 0.917 \\
\hline Acute fatigue & $571(33.4)$ & $432(33.0)$ & $110(32.9)$ & $29(43.9)$ & 0.187 \\
\hline Nausea and/or vomiting & $732(42.8)$ & $578(44.1)$ & $123(36.8)$ & $31(47.0)$ & 0.043 \\
\hline Diaphoresis & $561(32.8)$ & $448(34.2)$ & $93(27.8)$ & $20(30.3)$ & 0.081 \\
\hline \multicolumn{6}{|l|}{ First ECG, n (\%) } \\
\hline Non-ischaemic & $1515(88.5)$ & $1187(90.5)$ & $282(84.4)$ & $46(69.7)$ & $<0.001$ \\
\hline Non-specific changes $\dagger$ & $196(11.5)$ & $124(9.5)$ & $52(15.6)$ & $20(30.3)$ & $<0.001$ \\
\hline \multicolumn{6}{|c|}{ Symptom onset to first hs-cTnT (hours), n (\%) } \\
\hline$<3$ & $182(10.6)$ & $150(11.4)$ & $25(7.5)$ & $7(10.6)$ & 0.109 \\
\hline $3.0-5.99$ & 609 (35.6) & $474(36.2)$ & $114(34.1)$ & $21(31.8))$ & 0.637 \\
\hline $6.0-11.99$ & 409 (23.9) & $287(21.9)$ & $100(29.9)$ & $22(33.3)$ & 0.002 \\
\hline $12.0-23.99$ & $224(13.1)$ & $177(13.5)$ & 35 (10.5) & $12(18.2)$ & 0.159 \\
\hline$>24$ & $287(16.8)$ & $223(17.0)$ & $60(18.0)$ & $4(6.1)$ & 0.054 \\
\hline
\end{tabular}

All values are presented as $n(\%)$ and median (IQR). P values are for comparisons across the three triage groups using the Pearson $\chi^{2}$ test or the Fisher exact test for categorical variables, and the Kruskal-Wallis test for continuous variables.

The median time interval between the hs-cTnT samplings of 0 and 1 hour was $65 \mathrm{~min}$ (IQR 60-70) with no difference across the groups.

*Includes atrial fibrillation, other arrhythmias, cardiomyopathies, cerebral stroke, heart failure or valvular disease.

†Non-specific changes in either the ST segment, T inversions, Q waves, atrial fibrillation or left/right bundle branch block of unknown clinical significance.

COPD, chronic obstructive pulmonary disease; CVD, cardiovascular disease; hs-cTnT, high-sensitivity cardiac troponin T.

addition, the likelihood ratios (LRs) were obtained for all three groups. The area under the receiver operating characteristic (ROC) curve was constructed to report the overall diagnostic accuracy, with two cut-off values to include the intermediate observation group. ${ }^{27} 28$
A few cases of missing hs-cTnT values (due to errors or haemolysis) were separately handled by imputation using the median of the non-missing values. This was only done for a missing 1-hour value if the values of 0 and 4 hours were less than $3 \mathrm{ng} / \mathrm{L}$ apart, or for a missing 0 -hour test if 
the remaining values were all below the limit of detection $(<5 \mathrm{ng} / \mathrm{L})$. IBM SPSS V.25.0 and STATA V.15.0 were used in the calculations.

The study is registered at ClinicalTrial.gov and is conducted in accordance with the STARD (Standards for Reporting of Diagnostic Accuracy Studies) guidelines ${ }^{27}$ (online supplementary table S1).

\section{Patient and public involvement}

This research was designed and conducted without patient involvement.

\section{RESULTS}

\section{Participants}

During the patient enrolment period, 11618 patients presented to the OAEOC with acute chest pain or other symptoms suggestive of AMI. After the initial clinical assessment by the GP, hs-cTnT measurements was not considered necessary for approximately 6500 patients (ACS not suspected), while an estimated 2000 patients were directly transferred to the hospital with a highly suspected ACS and hence not available for study enrolment.

All 3066 consecutive patients admitted to the observation unit at the OAEOC for cardiac troponins were potentially eligible for the study. Of these, 1750 patients were included in the study (figure 1). Thirty-nine patients were excluded from the final data analyses, and 90-day follow-up data were not collected for 88 patients (figure 1). The 4-hour hs-cTnT was not sampled from $102(6 \%)$ patients in need of hospital transfer during the

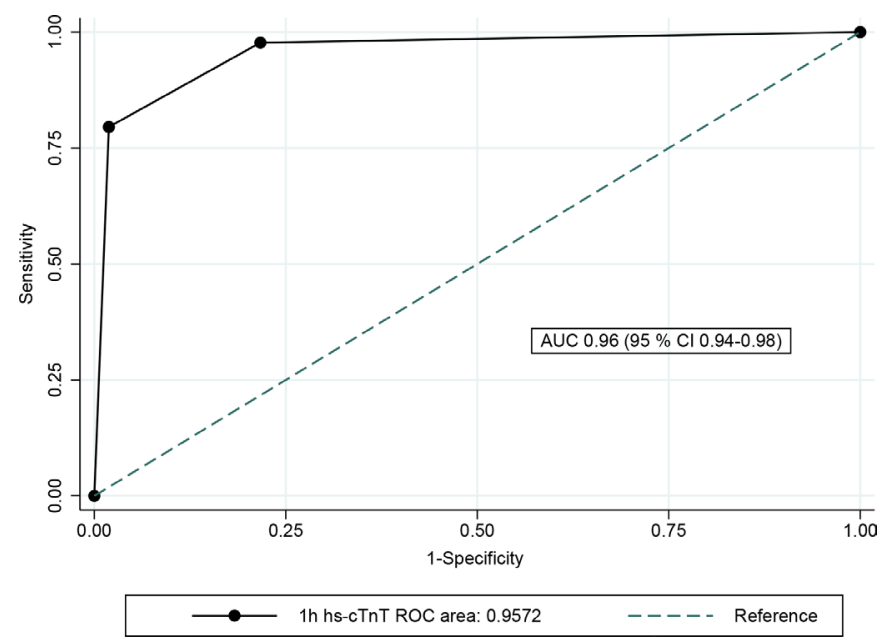

Figure 3 Overall diagnostic accuracy of the 0/1-hour algorithm for hs-cTnT. The overall diagnostic accuracy for AMI during the index episode was demonstrated by the area under the ROC curve at $96.0 \%$ (95\% Cl 0.94\% to $0.98 \%$ ). The AUC was achieved by using two cut-off values to include the observation group: (1) rule-in: sensitivity $45 / 61=0.74$ and specificity $(1310+319) / 1650=0.99$, (2) rule-out: sensitivity $(15+45) / 61=0.98$ and specificity: $1310 / 1650=0.79$. AMI, acute myocardial infarction; AUC, area under the curve; hs-cTnT, high-sensitivity cardiac troponin T; ROC, receiver operating characteristic. observation, 45 with an AMI, the remaining with other acute illnesses. These patients were not excluded from the study, and hospital documents were also collected for them.

\section{Characteristics of the study participants}

The study population $(\mathrm{n}=1711)$ had a median age of 56 (IQR 45-68) years, and $47.7 \%$ were women. The patients were categorised into either rule-out $(\mathrm{n}=1311,76.6 \%)$, rule-in $(n=66,3.9 \%)$ or observation group $(n=334,19.5$ $\%$ ) according to the 0/1-hour algorithm for hs-cTnT. The baseline characteristics of the patients are shown in table 1. A large proportion (29.9\%) of the patients were late presenters ( $>12$ hours duration of symptoms), and the rule-out group had significantly less comorbidity than the two other groups.

\section{AMI and hospitalisation}

Only $3.6 \%(61 / 1711)$ of the patients were adjudicated with an AMI diagnosis during the index episode: 1 patient in the rule-out group, 15 patients in the observation group and 45 among the rule-ins. The median age of patients with AMI was 65 years (IQR 55-73), 26 (42.6\%) of them were women. Sixty of the patients with AMI were hospitalised. Details regarding the hs-cTnT values among the patients with AMI are listed in online supplementary table S2.

In total, $13.2 \%(226 / 1711)$ of the patients were transferred to the hospital, $6.0 \%(79 / 1311)$ in the rule-out group, $27.2 \%$ (91/334) in the observation group and $84.8 \%(56 / 66)$ in the rule-in group. Among the hospitalised patients who did not have an AMI, 74 patients had at least one hs-cTnT value above the upper reference limit (online supplementary table S3).

\section{Applying the 0/1-hour algorithm}

The diagnostic performance of the 0/1-hour algorithm when applied in a primary care emergency setting is demonstrated in figure 2 . The safety in the rule-out group is substantiated by a high sensitivity of $98.4 \%$, an NPV of $99.9 \%$ and a negative LR of 0.02 . The rule-in accuracy has a high specificity of $98.7 \%$, a moderate PPV of 68.2 $\%$ and a positive LR of 58.0. The observation group in need of further tests received an indeterminate LR for AMI of 1.0. One of 1311 patients $(0.08 \%)$ in the rule-out group was wrongly classified. Details regarding misclassification by the $0 / 1$-hour algorithm are listed in online supplementary table S4.

The diagnostic performance of the 0/1-hour algorithm is also demonstrated by the ROC curve, constructed by two cut-off values defining the observation group between the rule-in group (sensitivity $73.8 \%$ and specificity $98.7 \%$ ) and the rule-out group (sensitivity $98.4 \%$ and specificity $79.4 \%$ ). This resulted in an area under the curve (AUC) of 0.96 (95\% CI 0.94 to 0.98 ) (figure 3 ). The total accuracy and overall efficacy was $79.2 \%$ and $80.5 \%$, respectively. 
Table 2 Prognostic performance of the 0/1-hour algorithm after 90 days

\begin{tabular}{|c|c|c|c|c|}
\hline Patients, n (\%) & AMI index* & Total AMI 90 days $†$ & Deaths 90 days & $\begin{array}{l}\text { AMI + deaths } 90 \\
\text { days } \neq\end{array}$ \\
\hline \multicolumn{5}{|l|}{ Disposition after OAEOC } \\
\hline Rule-out (n=1241) & $1(0.1)$ & $2(0.2)$ & $1(0.1)$ & $3(0.3)$ \\
\hline Primary care & 1 & 2 & 1 & 3 \\
\hline Hospital & 0 & 0 & 0 & 0 \\
\hline Observation $(n=320)$ & $15(4.5)$ & $19(5.7)$ & $4(1.3)$ & $22(6.9)$ \\
\hline Primary care & 0 & 3 & 1 & 4 \\
\hline Hospital & 15 & 16 & 3 & 19 \\
\hline Rule-in ( $n=62)$ & $45(68.2)$ & $45(68.2)$ & $4(6.4)$ & $45(68.2)$ \\
\hline Primary care & 0 & 0 & 0 & 0 \\
\hline Hospital & 45 & 45 & 4 & 45 \\
\hline Total $(\mathrm{N}=1623)$ & $61(3.6)$ & $66(4.1)$ & $9(0.6)$ & $70(4.3)$ \\
\hline
\end{tabular}

The patients were divided into the 0/1-hour algorithm classification and disposition after OAEOC discharge. Time to first incident of AMI is reported, including index episode, in addition to all-cause death the following 90 days. Follow-up data were not available for 2019 due to technical data-extraction reasons from the national registries, shortening the follow-up period for the 53 patients recruited to the study in October 2018.

${ }^{*} A M I$ at index admission: total $(N=1711)$; rule-out $(n=1311)$; observation $(n=334)$; rule-in $(n=66)$.

†lncluding AMI at index.

\#Five patients with AMI subsequently died (four in the rule-in group and one in the observation group) and hence were not counted twice. AMI, acute myocardial infarction; OAEOC, Oslo Accident and Emergency Outpatient Clinic.

\section{0-day prognostic performance}

During the first 90 days following admission to the observation unit, five new patients experienced an AMI, and there were in total nine deaths among the $1623 / 1711$ patients $(94.9 \%)$ consenting to linkage with the national registry (table 2). The total incidence of AMI or allcause death among the rule-out patients was $0.3 \%$ (the one death occurred on day 90 ). None of the 10 rule-in patients who were discharged home had an AMI or died the following 90 days, nor did the one false negative in the rule-out group.

\section{DISCUSSION}

Our study demonstrated that the 0/1-hour algorithm for hs-cTnT, when used in combination with clinical assessment and the ECG, safely rules out AMI, also in a lowprevalence setting outside of hospital. For the rule-out group, we found a high rule-out safety with an NPV of $99.9 \%$, a sensitivity of $98.4 \%$ and a very low 90 -day incidence of AMI or death (0.3\%). Our high NPV is comparable to previous hospital validation cohorts with NPVs exceeding $98 \% .^{13-16} 18$ For the rule-in group, the specificity is high $(98.7 \%)$, but with a moderate PPV of $68.2 \%$, as expected when a test is applied on a low-prevalence population. ${ }^{29}$ The AUC of $96.0 \%$ shows the overall diagnostic accuracy of the algorithm. In addition, a high efficacy has been demonstrated, with $80.5 \%$ of the patients assigned to either rule-out $(76.6 \%)$ or rule-in $(3.9 \%)$ by the algorithm. Also, as an LR-/+ below 0.1 or above 10.0 is considered strong evidence for ruling out or in a diagnosis,${ }^{30}$ our LR - of 0.02 and LR+ 58.0 reflect the high diagnostic performance of the algorithm.
Compared with the rule-out group, the patients assigned to the observation group $(19.5 \%)$ were older, had more comorbidity, higher baseline hs-cTnT values, and higher rates of AMI or death the following 90 days, which is probably why $27.2 \%$ of them were sent on to hospital, compared with $6.0 \%$ in the rule-out group. The LR of 1.0 in our observation group also reflects that the algorithm was not able to rule the patients in or out; hence, this group requires repeated hs-cTnT and further assessment. $^{12} 3031$

In our study, the majority of patients with AMI were late presenters and had a median age of 65 years, which is lower than the Norwegian average for patients with AMI (73.6 years). ${ }^{32}$ This is probably because early presenters with ongoing symptoms and elderly patients with several comorbidities were more likely to be considered as highrisk for ACS and directly hospitalised.

Recently, troponin assays, as well as hospital admissions for chest pain in a low-risk patient population, have been reported as examples of overuse of care. ${ }^{33}$ In our study, 21 of the rule-ins did not have an AMI. Ten of these patients were sent home with further management in primary care (table 2); none of them were readmitted with an AMI or died the following 90 days. The remaining 11 patients were hospitalised with other acute conditions that required hospitalisation (online supplementary table S4). Therefore, we do not think these 11 patients represent overuse of care, as the algorithm detects acute myocardial injury in addition to AMI. ${ }^{1234}$ It is also essential to recognise that the algorithm only rules out AMI and not unstable angina. ${ }^{1234}$ 
The algorithm performed well in our setting and could improve the prehospital assessment of patients with low-risk for ACS. Prehospital implementation of the 0/1-hour algorithm might also reduce crowding in the EDs and the need for hospitalisation of low-risk patients. Furthermore, accelerated rule-in in primary care will enable earlier hospital transfer for patients with atypical AMI (eg, women, diabetics and elderly patients). Further studies are warranted, investigating the cost-effectiveness of a prehospital implementation of the high-sensitivity 0/1-hour algorithm.

\section{Strengths and limitations}

Not including patients with highly suspected ACS provided a selected study population, which might be considered a limitation. On the other hand, this study aimed to validate the algorithm in a primary care emergency setting with a low prevalence population, complementary to previous hospital ED studies. It is essential that primary care clinics should never delay hospitalisation by offering repeated hs-cTnT sampling if an acute AMI is suspected. ${ }^{4}$ Accordingly, prehospital hs-cTnT sampling is only available at the OAEOC for patients considered low to moderately suspicious for ACS (online supplementary figure S1). The patients admitted to the observation unit comprise low-risk patients and patients with atypical symptoms such as acute dyspnoea without chest pain, acute fatigue and diaphoresis. Similar lowrisk patients are found among patients with chest pain in EDs in systems of care where patients primarily present directly to the hospital ED. However, as admission to the OAEOC observation unit is dependent on assessment by a GP, high-risk patients were identified and sent on to hospital prior to study enrolment, rendering a selected low-risk, low-prevalence study population. We consider our selected low-prevalence population a strength more than a limitation for the purposes of our study, and our results are probably generalisable to other primary care emergency settings with a capacity for short-term observation of low-risk patients.

Our 3.6\% AMI prevalence is low. The diagnostic performance of the algorithm is based on a limited number of events and calls for cautious interpretation of the numbers, especially the high LR+ (58.0) and the excellent NPV of $99.9 \% .^{29}$

The study did not evaluate the 0/1-hour algorithm for patients with chronic kidney dysfunction stages IV and V (estimated glomerular filtration rate of $<30 \mathrm{~mL}$ / $\left.\min / 1.73 \mathrm{~m}^{2}\right)$, as these patients were excluded from the final analyses. Furthermore, the informed consent form was only available in Norwegian and English, preventing the recruitment of 169 patients due to language barriers. By having the consent form available in additional languages, the population studied might have been more representative. The study also lacks information about the patients' country of origin.

Patients were approached for study enrolment by the regular nursing staff continuously, including holidays, weekends and nights, thus reducing potential selection bias. Still, 1316 of the patients admitted for prehospital hs-cTnT measurements were not included in the study (figure 1). Approximately half of them were missed due to time limitations $(n=111)$, staff errors $(n=254)$ and other not reported causes $(\mathrm{n}=264)$, as is to be expected in a study without additional designated research staff. Apart from missed inclusions due to language barriers, we do not think the non-included patients impact on the generalisability of our results.

The cardiologists did not adjudicate patients who were discharged home from the OAEOC. It was not ethical or feasible to offer these patients additional tests at the hospital. The resulting uncertainty concerning the final diagnosis is a limitation. Nonetheless, the incidence of AMI and death during the subsequent 90 days were very low in the rule-out group. In addition, the 1-hour study samples were available for the treating GP to avoid a delay in hospital transfer for patients with a significant 1-hour increase. Accordingly, the 1-hour sample was also available in the records used by the adjudication committee.

Finally, since this study is an observational study, it only demonstrates how the 0/1-hour algorithm might perform if implemented in a primary care setting. An implementation study investigating how the algorithm actually performs in real-life practice outside of hospital EDs is warranted.

\section{CONCLUSION}

The 0/1-hour algorithm for hs-cTnT seems safe, effective and applicable for implementation in a low-prevalence population for ACS outside of hospital when used in combination with clinical assessment and ECG. This might enable a faster assessment of patients presenting with acute non-specific chest pain in a primary care emergency setting, reduce unnecessary hospitalisations and hence decrease healthcare expenditure.

Twitter Tonje R Johannessen @tonjerj

Acknowledgements The authors are indebted to the patients participating in this study, the staff at the observation unit at the 0slo Accident and Emergency Outpatient Clinic and the laboratory technicians at Oslo University Hospital, Ullevaal. The authors also express their gratitude to Dr Arne Westheim, Dr Anne Kathrine Nore, Dr Kristin Moberg Aakre, Adjoa A. Badu Antwi, Tone Klette Boehler and Linda Andersen. In addition, the authors acknowledge the support from the Norwegian Cardiovascular Disease Registry and the TSD facilities at the University of Oslo, IT Department.

Contributors All authors have contributed in the conception, drafting and revision of the article, and approved the final version for publication.

Funding TRJ received funding from the Norwegian Research Fund for General Practice, the Norwegian Committee on Research in General Practice and the Norwegian Medical Association's Fund for Quality Improvement and Patient Safety. The funders of the study had no role in study design, data collection, data analysis, data interpretation or writing of the report.

Competing interests DA has received speaker's honoraria and consultancy fees from Amgen, Astra Zeneca, Bayer Healthcare, BMS, Boehringer-Ingelheim, Merck, Novartis, Pfizer and Sanofi-Aventis, and research grants from the institution by Medtronic and BMS. SH has received speaker's honoraria and consultancy fees 
from Amgen, Astra Zeneca, Bayer Healthcare, BMS, Boehringer-Ingelheim, Merck, Novartis, Pfizer and Sanofi-Aventis, and has no conflicts in relation to this study.

Patient consent for publication Not required.

Ethics approval Written informed consent was obtained from all participants. The study was carried out according to the principles of the Declaration of Helsinki and was approved by the local ethics committees (Regional Committee North for Medical and Health Research Ethics (number 2016/1241) and the Oslo University Hospital Information Security and Privacy Office (number 2016/13308)).

Provenance and peer review Not commissioned; externally peer reviewed.

Data availability statement Data may be made available upon reasonable request.

Open access This is an open access article distributed in accordance with the Creative Commons Attribution Non Commercial (CC BY-NC 4.0) license, which permits others to distribute, remix, adapt, build upon this work non-commercially, and license their derivative works on different terms, provided the original work is properly cited, appropriate credit is given, any changes made indicated, and the use is non-commercial. See: http://creativecommons.org/licenses/by-nc/4.0/.

\section{ORCID iDs}

Tonje R Johannessen http://orcid.org/0000-0001-9368-1471

Odd Martin Vallersnes http://orcid.org/0000-0003-1213-392X

Sigrun Halvorsen http://orcid.org/0000-0001-7561-7644

Ibrahimu Mdala http://orcid.org/0000-0002-5204-1934

Dan Atar http://orcid.org/0000-0003-1513-8793

\section{REFERENCES}

1 Thygesen K, Alpert JS, Jaffe AS, et al. Third universal definition of myocardial infarction. J Am Coll Cardiol 2012;60:1581-98.

2 Roffi M, Patrono C, Collet J-P, et al. 2015 ESC guidelines for the management of acute coronary syndromes in patients presenting without persistent ST-segment elevation: Task force for the management of acute coronary syndromes in patients presenting without persistent ST-segment elevation of the European Society of cardiology (ESC). Eur Heart J 2016;37:267-315.

3 Burman RA, Zakariassen E, Hunskaar S. Management of chest pain: a prospective study from Norwegian out-of-hours primary care. $B M C$ Fam Pract 2014;15:51.

4 Beygui F, Castren M, Brunetti ND, et al. Pre-Hospital management of patients with chest pain and/or dyspnoea of cardiac origin. A position paper of the acute cardiovascular care association (ACCA) of the ESC. Eur Heart J Acute Cardiovasc Care 2020;9:59-81.

5 Harskamp RE, Laeven SC, Himmelreich JC, et al. Chest pain in general practice: a systematic review of prediction rules. BMJ Open 2019;9:e027081.

6 Rasmussen MB, Stengaard C, Sørensen JT, et al. Predictive value of routine point-of-care cardiac troponin T measurement for prehospital diagnosis and risk-stratification in patients with suspected acute myocardial infarction. Eur Heart J Acute Cardiovasc Care 2019;8:299-308.

7 van Dongen DN, Tolsma RT, Fokkert MJ, et al. Pre-Hospital risk assessment in suspected non-ST-elevation acute coronary syndrome: a prospective observational study. Eur Heart $J$ Acute Cardiovasc Care 2020;9:5-12.

8 Stopyra JP, Snavely AC, Scheidler JF, et al. Point-Of-Care troponin testing during ambulance transport to detect acute myocardial infarction. Prehosp Emerg Care 2020:1-9.

9 Alghamdi A, Body R. BET 1: Prehospital cardiac troponin testing to 'rule out' acute coronary syndromes using point of care assays. Emerg Med J 2018;35:572-4.

10 Reichlin T, Hochholzer W, Bassetti S, et al. Early diagnosis of myocardial infarction with sensitive cardiac troponin assays. N Engl $J$ Med 2009;361:858-67.

11 Giannitsis E, Becker M, Kurz K, et al. High-Sensitivity cardiac troponin $\mathrm{T}$ for early prediction of evolving non-ST-segment elevation myocardial infarction in patients with suspected acute coronary syndrome and negative troponin results on admission. Clin Chem 2010;56:642-50.

12 Westermann D, Neumann JT, Sörensen NA, et al. High-Sensitivity assays for troponin in patients with cardiac disease. Nat Rev Cardiol 2017;14:472-83.

13 Reichlin T, Schindler C, Drexler B, et al. One-Hour rule-out and rule-in of acute myocardial infarction using high-sensitivity cardiac troponin T. Arch Intern Med 2012;172:1211-8.

14 Reichlin T, Twerenbold R, Wildi K, et al. Prospective validation of a 1-hour algorithm to rule-out and rule-in acute myocardial infarction using a high-sensitivity cardiac troponin T assay. CMAJ 2015;187:E243-52.

15 Pickering JW, Greenslade JH, Cullen L, et al. Assessment of the European Society of cardiology 0-Hour/1-Hour algorithm to Rule-Out and Rule-In acute myocardial infarction. Circulation 2016;134:1532-41.

16 Mueller C, Giannitsis E, Christ M, et al. Multicenter evaluation of a 0-Hour/1-Hour algorithm in the diagnosis of myocardial infarction with high-sensitivity cardiac troponin T. Ann Emerg Med 2016:68:76-87.

17 Mokhtari A, Borna C, Gilje P, et al. A 1-h Combination Algorithm Allows Fast Rule-Out and Rule-In of Major Adverse Cardiac Events. J Am Coll Cardiol 2016:67:1531-40.

18 Twerenbold R, Neumann JT, Sörensen NA, et al. Prospective validation of the 0/1-h algorithm for early diagnosis of myocardial infarction. J Am Coll Cardiol 2018;72:620-32.

19 Statistics Norway. Table 01222 [internet], 2019. Available: www.ssb. no/en/statbank/table/01222 [Accessed 13 Jan 2020].

20 Blinkenberg J, Pahlavanyali S, Hetlevik Øystein, et al. General practitioners' and out-of-hours doctors' role as gatekeeper in emergency admissions to somatic hospitals in Norway: registrybased observational study. BMC Health Serv Res 2019;19:568.

21 Norwegian Institute of Public Health. Norwegian cardiovascular disease registry: Norwegian Institute of public health, 2012. Available: https://www.fhi.no/en/hn/health-registries/cardiovasculardisease-registry/ [Accessed 23 Mar 2020].

22 World Health Organisation. International statistical classification of diseases and related health problems 10th revision (ICD-10. 15th edn. Geneva, Switzerland: WHO Press, 2016.

23 Egeland GM, Sundvor V, Igland J, et al. Opportunities for diabetes research using the Norwegian cardiovascular disease registry. Nor Epidemiol 2013;23:95-100.

24 Giannitsis E, Kurz K, Hallermayer K, et al. Analytical validation of a high-sensitivity cardiac troponin T assay. Clin Chem 2010;56:254-61.

25 Saenger AK, Beyrau R, Braun S, et al. Multicenter analytical evaluation of a high-sensitivity troponin T assay. Clin Chim Acta 2011:412:748-54.

26 Li A, Brattsand G. Stability of serum samples and hemolysis interference on the high sensitivity troponin T assay. Clin Chem Lab Med 2011:49:335-6.

27 Bossuyt PM, Reitsma JB, Bruns DE, et al. Stard 2015: an updated list of essential items for reporting diagnostic accuracy studies. BMJ 2015:351:h5527.

28 Baduashvili A, Guyatt G, Evans AT. Roc Anatomy-Getting the most out of your diagnostic test. J Gen Intern Med 2019;34:1892-8.

29 Brenner H, Gefeller O, sensitivity Vof. Variation of sensitivity, specificity, likelihood ratios and predictive values with disease prevalence. Stat Med 1997:16:981-91.

30 Deeks JJ, Altman DG. Diagnostic tests 4: likelihood ratios. BMJ 2004:329:168-9.

31 Nestelberger T, Wildi K, Boeddinghaus J, et al. Characterization of the observe zone of the ESC 2015 high-sensitivity cardiac troponin $\mathrm{Oh} / \mathrm{h}$-algorithm for the early diagnosis of acute myocardial infarction. Int J Cardiol 2016;207:238-45.

32 Sulo G, Igland J, Vollset SE, et al. Trends in incident acute myocardial infarction in Norway: an updated analysis to 2014 using national data from the CVDNOR project. Eur J Prev Cardiol 2018:25:1031-9.

33 Scott IA. Audit-based measures of overuse of medical care in Australian hospital practice. Intern Med J 2019;49:893-904.

34 Jaffe AS, White H, Injury R-IM. Ruling-In myocardial injury and Ruling-Out myocardial infarction with the European Society of cardiology 1-hour algorithm. Circulation 2016;134:1542-5. 